(c) 2021 Universidad Nacional Autónoma de México, Facultad de Estudios Superiores Zaragoza.

Este es un artículo Open Access bajo la licencia CC BY-NC-ND (http://creativecommons.org/licenses/by-nc-nd/4.0/).

TIP Revista Especializada en Ciencias Químico-Biológicas, 24: 1-10, 2021.

https://doi.org/10.22201/fesz.23958723e.2021.302

\title{
Tratamiento a largo plazo con campos electromagnéticos en un modelo animal de la enfermedad de Parkinson
}

\author{
Idalia Medina-Salazar ${ }^{1}$, Jacqueline Moreno-Fitz ${ }^{1}$, Grecia Jiménez ${ }^{1}$, \\ Noé Morales ${ }^{1}$, Monserrat Pizarro ${ }^{1}$, David Elías-Viñas ${ }^{\dagger 2}$ y Leticia Verdugo-Díaz ${ }^{1 *}$ \\ ${ }^{1}$ Laboratorio de Bioelectromagnetismo, Departamento de Fisiología, Facultad de Medicina, UNAM, \\ Circuito Escolar, Ciudad Universitaria, Alcaldía Coyoacán 04510, Ciudad de México, México. \\ ${ }^{2}$ Departamento de Ingeniería Eléctrica, Sección de Bioelectrónica, CINVESTAV, IPN, Alcaldía Gustavo A. \\ Madero 07360, Ciudad de México, México. E-mail: *leticia@unam.mx
}

\begin{abstract}
RESUMEN
La enfermedad de Parkinson (EP) es un padecimiento neurodegenerativo que afecta las neuronas de la sustancia negra pars compacta $(\mathrm{SNc})$, provocando alteraciones motoras y no motoras. En la actualidad para el tratamiento de pacientes con EP se aplica la Estimulación con Campos Electromagnéticos (CEM), observándose cierta mejoría clínica. Sin embargo, su mecanismo terapéutico subyacente sigue sin ser dilucidado. Los estudios en modelos animales de la EP se han realizado por periodos cortos de estimulación con CEM. Por lo tanto, el objetivo del presente estudio fue investigar los cambios en el comportamiento motor de ratas Wistar con hemiparkinson, tratadas con CEM de $60 \mathrm{~Hz}, 2$ horas diariamente durante 6 meses. Mensualmente se realizaron evaluaciones de conducta: rotación inducida por apomorfina, campo abierto, laberinto elevado en cruz y barra de equilibrio. Al finalizar el tratamiento se contó el número de neuronas dopaminérgicas en la SNc y se estimó su grado de pérdida. Los animales lesionados que recibieron CEM tuvieron una ganancia de peso similar a los animales control. El tratamiento redujo la conducta de giro y mejoró el equilibrio, sin cambios significativos en la sobrevida de las neuronas dopaminérgicas de la SNc. Estos resultados apoyan el uso de los CEM de $60 \mathrm{~Hz}$ como una terapia complementaria que puedan ayudar a mejorar los síntomas motores de la enfermedad. Palabras clave: ansiedad, equilibrio, hemiparkinson, locomoción, neuronas dopaminérgicas, rata.
\end{abstract}

\section{Long-term treatment with electromagnetic fields in an animal model of Parkinson's disease}

\begin{abstract}
Parkinson's disease (PD) is a neurodegenerative disease that causes motor and non-motor alterations induced by the loss of neurons of substance nigra pars compacta ( $\mathrm{SNc}$ ) In clinical therapy, the use of pharmacological treatments to improve symptoms is commonly used. Recently, non-pharmacological techniques are being developed. Such is the case for Electromagnetic Field Stimulation (EMF). Although this treatment has already been used in PD patients, its underlying therapeutic mechanism remains unclear. Studies in animal models of PD have been performed but only for short periods of stimulation with EMF. This study aimed to investigate motor behavior in hemiparkinsonian Wistar rats treated with EMF of $60 \mathrm{~Hz}(2.4 \mathrm{mT}) 2$ hours daily for six months. Behavioral evaluations such as apomorphine-induced rotation, open field test, elevated cross maze, and balance beam were performed monthly. Also, immunoreactive dopaminergic neurons were counted, and their degree of loss was estimated. The main results showed that lesioned animals treated with EMF, did not differ in weight gain compared to control animals. Furthermore, magnetic treatment reduced turning behavior and improved balance, without significant changes in the survival of SNc dopaminergic neurons. These results support the use of EMF as an alternative therapy that could help to improve the motor symptoms of PD.
\end{abstract}

Keywords: anxiety, dopaminergic neurons, equilibrium, hemiparkinson, locomotion, rat.

Artículo recibido el 10 de octubre del 2020.

Artículo aceptado el 13 de marzo del 2021. 


\section{INTRODUCCIÓN}

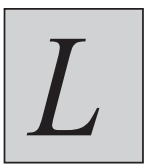

a Enfermedad de Parkinson (EP) es uno de los trastornos neurodegenerativos más frecuentes en el mundo y se produce como resultado de la muerte de las neuronas dopaminérgicas en la sustancia negra pars compacta (SNc). Este padecimiento se caracteriza por presentar ciertos trastornos motores, como el temblor en reposo, bradicinesia y trastornos de la marcha (Miller, SuarezIglesias, Seijo-Martínez \& Ayán, 2020). En etapas avanzadas pueden surgir síntomas cognitivos, conductuales, alteraciones autonómicas y psiquiátricas; particularmente demencia y depresión (Jankovic, 2008).

El uso de dopamina es un tratamiento eficaz, utilizado para disminuir los síntomas de la EP; sin embargo, al usarlo durante largo plazo se crea resistencia al medicamento. Un $24 \%$ de los pacientes tiene fluctuaciones motoras, con un promedio de 5.5 episodios "off" diarios, de 44 minutos con un total diario de siete horas "off”" (Fàbregues, Gironell, RossellóJiménez \& Regueras, 2017). Además, pueden aparecer con mayor frecuencia síntomas psiquiátricos como alucinaciones, apatía o ansiedad. En consecuencia, se han investigado otros tratamientos para la EP como: las de tipo quirúrgico (palidotomía), las estimulaciones invasivas (estimulación cerebral profunda) y no invasivas (estimulación magnética transcraneal) (Shukla \& Vaillancourt, 2014). La terapia electromagnética representa una opción prometedora, ya que promueve una mejoría en los síntomas motores y no motores (Rektorová \& Anderkova, 2017). La terapia electromagnética se define como el uso de campos electromagnéticos variables en el tiempo, que pueden inducir una corriente suficientemente fuerte para estimular los tejidos adyacentes (Chervyakov, Chernyavsky, Sinitsyn \& Piradov, 2015).

En el 2016, se publicó un meta-análisis sobre el uso de la estimulación magnética transcraneal para el tratamiento de problemas motores de la EP, en esa publicación fueron seleccionados 21 estudios y en ellos se concluye que esta terapia puede tener moderados efectos positivos en los pacientes que la padecen (Shukla, et al., 2016). En contraste, estudios epidemiológicos han reportado una interacción de la exposición a los CEM con un aumento en la incidencia de la EP (Gervasi, Murta, Decarli \& Russo, 2019; Gunnarsson \& Bodin, 2019). La divergencia entre los resultados publicados sobre el efecto de los CEM y la EP implica que éstos aún no son concluyentes y que deben de ampliarse los estudios de exposiciones a largo plazo y con diversos parámetros de estimulación.

Los campos electromagnéticos (CEM) de extrema baja frecuencia pueden modificar a los neurotransmisores $\mathrm{y}$ la conducta (Chance et al., 1995); así como alterar la excitabilidad de la corteza (Ni \& Chen, 2015). La estimulación magnética por 21 días produjo un aumento en los niveles de las neurotrofinas y una mejora en las pruebas conductuales en un modelo animal de la Enfermedad de Huntington (Tasset et al., 2012). Sin embargo, es poco el conocimiento que se tiene sobre el comportamiento motor en modelos animales de la EP estimulados con los CEM. Umarao y colaboradores demostraron que en ratas lesionadas con hidroxidopamina y estimuladas durante una semana los efectos son benéficos en la conducta alimenticia, el equilibrio y la postura (Umarao, Bose, Bhattacharyya, Kumar \& Jain, (2016). Además, un aumento en la expresión cerebral de diferentes factores de crecimiento ha sido reportado como resultado de la estimulación electromagnética (Lee et al., 2013), y han sido relacionados con la neuroprotección y supervivencia de las neuronas (Arias-Carrión et al., 2004).

En un estudio previo realizado en nuestro laboratorio, se llevaron a cabo, en ratas con el modelo de hemiparkinson, pruebas de conducta motora después de 2 meses de estimulación ( 2 h/día) con CEM de $60 \mathrm{~Hz}$. Los resultados obtenidos mostraron cambios benéficos en la locomoción, el equilibrio y reducción de los parámetros relacionados con la ansiedad (Moreno-Fitz, Medina-Salazar, Chávez-Hernández, Elías-Viñas \& Verdugo-Díaz, 2015). Sin embargo, con dos meses de estimulación varios de los parámetros conductuales solamente mostraron una tendencia a la recuperación. Por lo que en el presente estudio la incógnita fue si con un tiempo de estimulación mayor, sería más evidente la mejoría motora. El propósito de este estudio fue describir los efectos a largo plazo (6 meses), con el tratamiento de CEM, en la conducta motora y su relación con la pérdida de neuronas dopaminérgicas en la $\mathrm{SNc}$ de ratas Wistar con hemiparkinson.

\section{Materiales Y MÉTOdos}

Se utilizaron 44 ratas macho de la estirpe Wistar, con peso de 180-200 g. Las ratas tuvieron tres días de adaptación a las condiciones del laboratorio. Se alojaron dos ratas por jaula de acrílico $(16 \times 37 \times 18 \mathrm{~cm})$ en condiciones de temperatura $(21$ $\left.23^{\circ} \mathrm{C}\right)$, ciclos alternos de luz/oscuridad de 12 horas (07:00 encendido, 19:00 apagado), comida y agua a libre demanda. Los animales fueron pesados semanalmente. Todos los procedimientos fueron previamente aprobados por el comité de ética e investigación de la Facultad de Medicina de la UNAM de acuerdo con la Norma Oficial Mexicana (NOM062-ZOO-1999).

\section{Lesión con 6-hidroxidopamina (6-OHDA)}

Los animales se anestesiaron con ketamina, xilacina y acepromacina (66 mg, $0.26 \mathrm{mg}$ y $1.3 \mathrm{mg} / \mathrm{Kg}$ de peso del animal respectivamente) vía intraperitoneal. Se colocaron en un estereotáxico para ratas (Stoeling) y se localizó el sitio de inyección de acuerdo con las coordenadas $-3.5 \mathrm{~mm}$ anteroposterior a Bregma, $-1.5 \mathrm{~mm}$ lateral de la línea media y $-8.8 \mathrm{~mm}$ ventral de la superficie del cráneo (AriasCarrión et al., 2004). Se aplicaron $4 \mu \mathrm{L}$ de solución con 
6-hidroxidopamina (6-OHDA $2 \mu \mathrm{g} / \mu \mathrm{L}$ en solución de $\mathrm{NaCl}$ al $0.9 \%$ más ácido ascórbico al $0.5 \%$ ). Para los animales con falsa lesión (Sham) sólo se inyectó el vehículo.

\section{Diseño experimental}

Para evaluar el grado de denervación nigroestriatal, se realizó la prueba de giros inducidos por apomorfina 10 días después de la cirugía. Sólo se seleccionaron aquellos animales que mostraron una tasa constante de giro con más de 150 giros/ $30 \mathrm{~min}$ contralateral al lado lesionado (Truong, Allbutt, Kassiou \& Henderson, 2006). Los animales se dividieron en los siguientes grupos: ratas control sin tratamiento $(\mathrm{C}, \mathrm{n}=6)$, control con estimulación electromagnética $(C+C E M, n=6)$, animales con lesión simulada (Sham, $n=6$ ), Sham con CEM $($ Sham + CEM, $n=6$ ), lesionados con 6-OHDA (6-OHDA, $\mathrm{n}=10)$ y lesionados con CEM (6-OHDA + CEM, $\mathrm{n}=10)$. En la Figura 1 se muestra un diagrama del diseño experimental. El tratamiento con CEM se realizó diariamente 5 días a la semana de lunes a viernes, durante 6 meses.

\section{Exposición al campo electromagnético de frecuencia extremadamente baja (CEM)}

La exposición al CEM se aplicó con un dispositivo previamente utilizado (Moreno-Fitz et al., 2015). El CEM se generó con un par de bobinas en configuración Helmholtz de $30 \mathrm{~cm}$ de diámetro interno, compuestas de alambre de cobre (350 vueltas) diseñadas y construidas en el Centro de Investigación y Estudios Avanzados, IPN (Ciudad de México). Las bobinas se conectaron en paralelo a un transformador ajustable de 120 V (Staco Energy Products). Se acopló un osciloscopio (Tecktronix $5103 \mathrm{~N}$ ) al sistema para monitorear la forma sinusoidal de la onda magnética con frecuencia de $60 \mathrm{~Hz}$. La amplitud de la densidad del flujo magnético fue de $2.4 \mathrm{mT}$, que se midió con un medidor manual Gauss/Tesla (Alpha Lab). Estos parámetros son comúnmente utilizados por diversos aparatos de magnetoterapia (Belyaev et al., 2016; Mattsson \& Simko, 2019). La temperatura en el interior de las cámaras de exposición fue de $23.4 \pm 0.4{ }^{\circ} \mathrm{C}$ (Hygro-termometer Extech Instruments, Waltham, MA), este parámetro se mantuvo constante durante las 2 horas de estimulación. La exposición al CEM se realizó siempre entre las 9:00 y 13:00 h.

\section{Pruebas conductuales}

Se realizaron cuatro pruebas conductuales previas a la estimulación con el CEM (pre-CEM) y cada 30 días después de la estimulación (post-CEM). Las pruebas se realizaron entre las 10:00 y 15:00 h.

\section{Giros inducidos por apomorfina}

La prueba de giros se utiliza para verificar el grado del daño provocado por la lesión con 6-OHDA (Allbutt \& Henderson, 2007) y se llevó a cabo colocando cada rata en un cilindro de plástico (diámetro de $40 \mathrm{~cm}$ y $20 \mathrm{~cm}$ de alto) posterior a la administración intraperitoneal de $0.2 \mathrm{mg} / \mathrm{Kg}$ de clorhidrato de apomorfina (Sigma), disuelto en una solución salina de ascorbato al $0.1 \%$. Después de 5 minutos de haber aplicado la inyección, se contó durante 30 minutos el número total de giros hechos hacia ambos lados y se reportó como el número de giros contralaterales menos los ipsilaterales respecto a la lesión (Truong et al., 2006). Esta prueba solo se efectuó en animales con falsa lesión y lesionados.

\section{Barra de equilibrio}

Esta prueba es útil para valorar los déficits de locomoción, al medir la bradicinesia, el balance y la inestabilidad postural en modelos animales de la EP, con base en el tiempo que tardan en atravesar la barra (Allbutt \& Henderson, 2007). Los animales se colocaron en el extremo de una barra de madera de $1 \mathrm{~cm}$ de grosor y $100 \mathrm{~cm}$ de largo, elevada a $80 \mathrm{~cm}$ por encima del piso. La jaula que habitaba la rata se colocó en el otro extremo de la barra, como estímulo para que la cruzara. El tiempo que tardó en atravesarla, se midió con un cronómetro, siendo en el experimento el máximo permitido de ejecución de 2 minutos. Previo a esta prueba, se entrenó a los animales para que reconocieran la barra. El valor del tiempo promedio considerado para cada prueba fue de 4 recorridos.

\section{Laberinto elevado en cruz}

La prueba del laberinto elevado se estableció para explorar la ansiedad de los roedores (Horii, McTaggart \& Kawaguchi, 2018) y consistió en colocar al animal en el centro del laberinto en forma de cruz, que tiene 2 de sus brazos sin paredes (brazos abiertos) y 2 con muros (brazos cerrados), elevado a $50 \mathrm{~cm}$ del

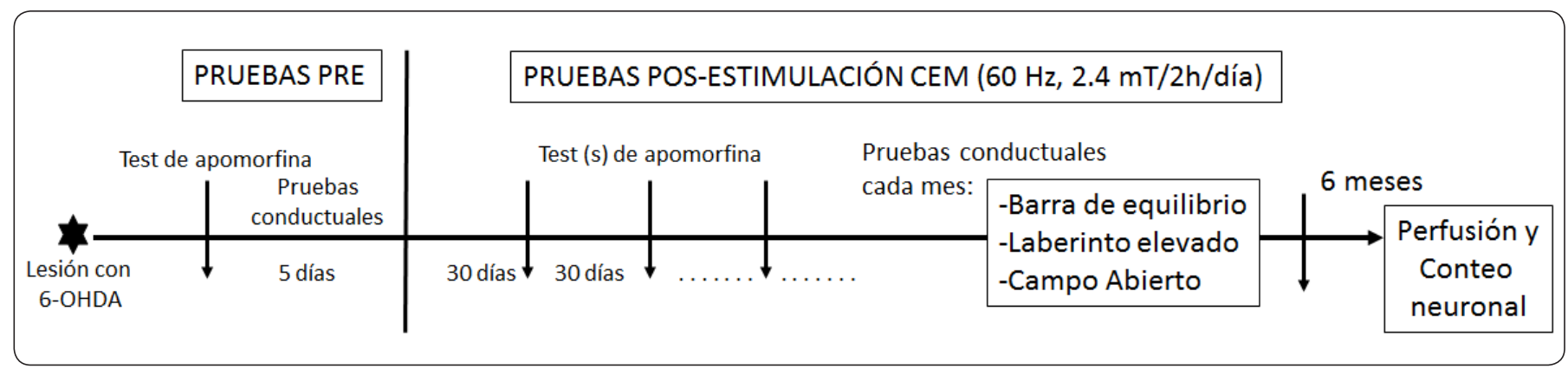

Figura 1. Diseño experimental. Elaboración personal. 
suelo. El comportamiento de los animales fue videograbado durante 5 minutos. Los parámetros evaluados en brazos abiertos y en cerrados fueron: número de entradas, tiempo de permanencia, la proporción de entradas y el número de heces (Schmitt \& Hiemke, 1998).

\section{Campo abierto}

Esta prueba se utiliza para medir la locomoción, conductas depresivas y de ansiedad (Picazo et al., 2009). El experimento consistió en videograbar por 15 minutos el comportamiento de la rata, al colocarla en el centro de una caja de acrílico negro (90 cm x $90 \mathrm{~cm}$ x $30 \mathrm{~cm}$ de altura), dividida por líneas en cuadrados de 15 x $15 \mathrm{~cm}$. En esta evaluación se registraron los siguientes datos: distancia total recorrida en centímetros, tiempo de inmovilidad, número de toques con las patas delanteras en las paredes laterales y número de heces (Schmitt \& Hiemke, 1998). Estos parámetros nos reflejan la ansiedad del animal por buscar una salida, la cual incrementa en los modelos de hemiparkinson.

\section{Inmunohistoquímica y conteo neuronal}

Al final de los 6 meses de estimulación, los animales fueron profundamente anestesiados con pentobarbital sódico (35 $\mathrm{mg} / \mathrm{Kg}$ ) y perfundidos trascardialmente con solución salina tamponada con fosfato ( $\mathrm{PBS}, 0.02 \mathrm{M}, \mathrm{pH}=7.2$ ) seguido de una solución al 4\% de paraformaldehído en PBS. Los cerebros se extrajeron y se crio-conservaron para su posterior tratamiento. Se realizaron cortes coronales con un espesor de $40 \mu \mathrm{m}$ con criostato de la zona del estriado y de SNc (1320, Ernest Leitz). Estos fueron tratados con el anticuerpo policlonal contra tirosina hidroxilasa (TH, 1:2000, Chemicon $A B$ 152) y revelados de acuerdo con las indicaciones de los proveedores del MACH 1 Universal, HRP-Polymer kit (Rabbit IgG, Elite pK-6101).

Para cuantificar a las neuronas inmunorreactivas a $\mathrm{TH}$, los límites de la SNc se establecieron de acuerdo con el atlas estereotáxico del cerebro de rata (desde -5.20 hasta $-6.30 \mathrm{~mm}$ respecto a Bregma; Paxinos \& Watson, 2005). Las imágenes fueron captadas por una cámara (Evolution VF Cooled Color Camera Medica Cybernetics) adaptada a un microscopio (Olympus IX-71) con un aumento de 4X y 20X; una vez adquiridas, se analizaron con el programa Image ProPlus 6.0. El conteo se realizó manualmente en los cortes que cubrían la totalidad de la SNc (aproximadamente $800 \mathrm{~mm}$ ), con base en los siguientes criterios: célula con citoplasma completamente marcado, núcleo perfectamente definido y sin marca (GómezChavarín et al., 2013). El conteo neuronal lo realizaron tres investigadores independientes.

\section{Estadística}

Todos los resultados se presentan con la media aritmética \pm error estándar de la media (EEM) y con $\mathrm{p}<0.05$ de significancia estadística. Los datos conductuales obtenidos a través del tiempo fueron analizados con un análisis de varianza de dos vías (ANOVA) con múltiples comparaciones y el conteo de neuronas dopaminérgicas con ANOVA de una vía, mediante el programa GraphPad Prism v.6.

\section{ReSUltados}

Las ratas que recibieron inyección unilateral de 6-OHDA, mostraron pérdida significativa de células inmunomarcadas $(\mathrm{TH}+)$ en la SNc del lado lesionado, a diferencia de aquellas que habían recibido inyección del vehículo $(\mathrm{p}<0.05)$. El tratamiento con el CEM no afectó el número de neuronas dopaminérgicas en ninguno de los grupos estudiados (Tabla I). La Figura 2 muestra una microfotografía de un corte cerebral de una rata lesionada e imágenes representativas de la región de SNc donde se realizó el conteo.

Los giros inducidos por apomorfina se muestran en la Figura 3. Los animales con el modelo de hemiparkinson presentaron un aumento en el número de giros, sobre todo durante los tres primeros meses después de la lesión, valores que permanecieron elevados durante los siguientes meses del experimento. En Los animales lesionados que recibieron tratamiento con el CEM disminuyó este parámetro, desde el segundo mes $\left(\mathrm{F}_{6,11}=8.3, \mathrm{p}<0.001\right)$.

El peso de los animales se midió mensualmente, los resultados se ven en la Figura 4. Los animales lesionados con 6-OHDA mostraron menor ganancia de peso comparados con los animales control desde el primer mes, esto fue estadísticamente significativo a partir del tercer mes $\left(\mathrm{F}_{3,88}=23.63, \mathrm{p}<\right.$ 0.001). Los animales lesionados que recibieron tratamiento electromagnético no presentaron esa diferencia, por el contrario, el aumento de peso fue similar al de los animales control durante los 6 meses del experimento $(p>0.05)$.

\section{Efectos del tratamiento con el CEM en conductas motoras y equilibrio}

El promedio de tiempo que tardaron los animales en recorrer la barra de equilibrio durante los 6 meses del experimento

Tabla I. Neuronas dopaminérgicas TH+ de los grupos experimentales.

\begin{tabular}{|l|c|c|}
\hline \multicolumn{1}{|c|}{ Grupo } & $\begin{array}{c}\text { Hemisferio no } \\
\text { lesionado }\end{array}$ & $\begin{array}{c}\text { Hemisferio } \\
\text { lesionado }\end{array}$ \\
\hline Control & $1484 \pm 161$ & $1191 \pm 14$ \\
\hline C+CEM & $994 \pm 262$ & $1039 \pm 455$ \\
\hline Sham & $1445 \pm 62$ & $1523 \pm 107$ \\
\hline Sham+CEM & $1407 \pm 357$ & $1448 \pm 382$ \\
\hline 6-OHDA & $1179 \pm 215$ & $339 \pm 52 *$ \\
\hline 6-OHDA+CEM & $1621 \pm 226$ & $458 \pm 253 *$ \\
\hline
\end{tabular}

Promedio \pm EEM. $* \mathrm{p}<0.05$ versus el hemisferio no lesionado. 


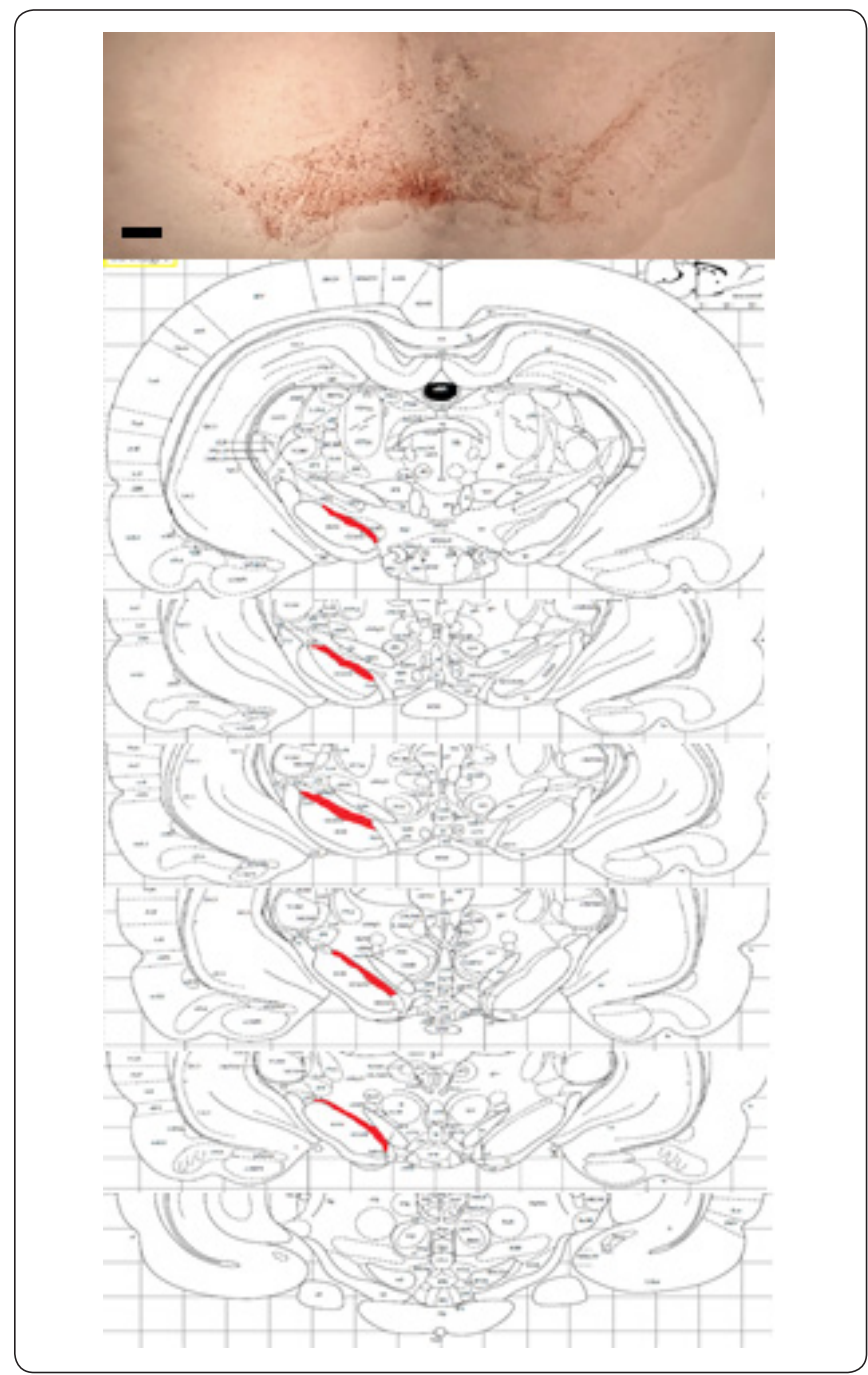

Figura 2. Lesión con 6-OHDA en el hemisferio izquierdo. A. Microfotografía representativa de inmunodetección de TH en un corte coronal de cerebro de rata con 6-OHDA, a nivel de la sustancia negra pars compacta $(\mathrm{SNc})($ barra $=100 \mathrm{~mm})$. B. Secciones representativas del atlas correspondiente a la zona de la SNc donde se realizaron los conteos neuronales. Elaboración personal.

se muestra en la Figura 5. Los animales con lesión y sin tratamiento, a los 5 y 6 meses, aumentaron el tiempo de recorrido en forma significativa comparado con sus valores basales $(\mathrm{p}<0.05)$. El tratamiento electromagnético en animales con hemiparkinson, desde el primer mes de exposición produjo una reducción en el tiempo que recorrieron la barra. Esta mejoría en el equilibrio y la coordinación motora fue estadísticamente significativa a los 4, 5 y 6 meses de tratamiento, respecto a los animales lesionados no tratados $\left(\mathrm{F}_{6,111}=8.30, \mathrm{p}<0.05\right)$.

En la Figura 6 se muestra el porcentaje de ingreso a brazos abiertos respecto al número total de entradas en el laberinto

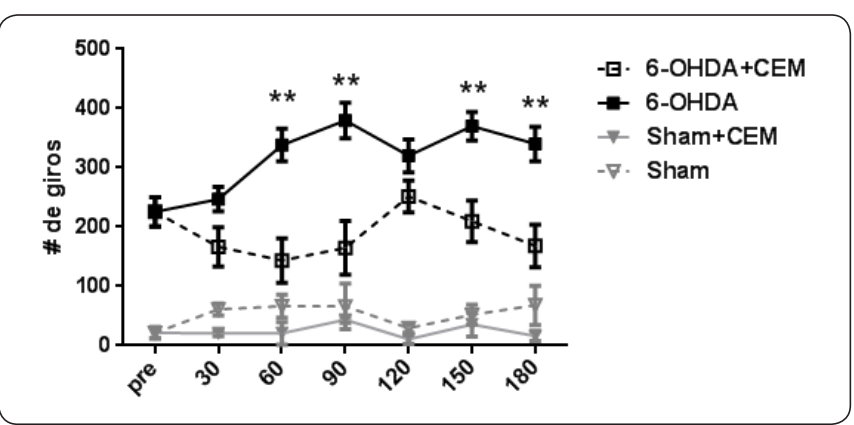

Figura 3. Giros contralaterales al lado lesionado. Promedio \pm EEM del número de giros inducidos por apomorfina antes de iniciar la estimulación (pre) y a los 30, 60, 90, 120, 150 y 180 días después de la estimulación con CEM. Los animales lesionados mostraron un aumento en el número de giros respecto a su nivel basal $(* p<0.05)$. Al comparar los animales con 6-OHDA contra los 6-OHDA + CEM, se observaron diferencias estadísticamente significativas a los 2, 3, 5 y 6 meses $(* * p<0.01)$. Elaboración personal.

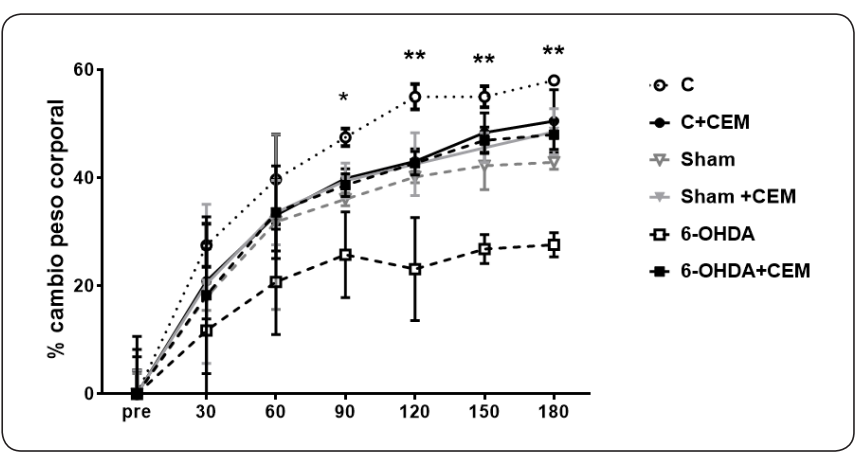

Figura 4. Ganancia de peso corporal. Promedio \pm EEM del porcentaje de ganancia de peso (g), con respecto al valor antes de iniciar la estimulación (pre), considerando este como cero. Los animales control son los que muestran un mayor porcentaje de aumento de peso a lo largo de los 6 meses del experimento. Este valor solo es significativamente mayor que el que presentan los animales lesionados (6-OHDA) a partir del tercer mes $\left({ }^{*} p<0.05\right.$, $* *$ p $<0.01)$. Elaboración personal.

elevado. Antes del tratamiento, los animales lesionados tuvieron un porcentaje de ingreso mayor que los animales control $(p<0.05)$. En cada mes graficado en la Figura 6, las últimas dos barras representan los valores de los animales lesionados y los lesionados+CEM. Durante tres meses, estos valores fueron similares en ambos grupos. A partir de los 120 días de estimulación, en los animales tratados con CEM disminuyeron significativamente el porcentaje de entradas comparados con las ratas en las que se inyectó 6-OHDA, que presentaron valores similares a los grupos con falsa lesión.

En la Tabla II se muestran los resultados de la prueba de campo abierto para los 2, 4 y 6 meses de estudio. Todos los grupos mostraron menor distancia recorrida a medida que 
transcurre el experimento $\left(\mathrm{F}_{6,153}=30.88, \mathrm{p}<0.001\right)$. Sin embargo, en los últimos meses esta disminución fue mayor en los grupos de animales lesionados comparados con los controles $(p<0.05)$. El movimiento vertical (toques en paredes) disminuyó en frecuencia a lo largo del experimento en todos los grupos. Esta reducción es significativamente mayor a los 6 meses para los grupos lesionados $(p<0.05)$.

\section{Discusión}

El presente estudio muestra que la exposición a campos magnéticos de extrema baja frecuencia, aplicados diariamente durante seis meses en un modelo de hemiparkinson en ratas, induce a cambios relacionados con el aumento de peso, el equilibrio y la ansiedad.

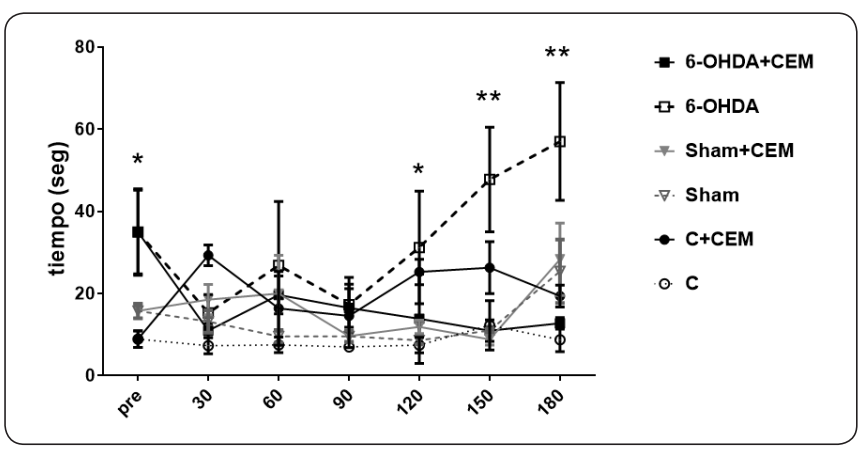

Figura 5. Barra de Equilibrio. Promedio \pm EEM del tiempo (segundos) que tardan los animales en recorrer la barra de equilibrio, antes de iniciar la estimulación (pre) y a los 30, 60, 90, 120, 150 y 180 días después de la estimulación con CEM. Durante las pruebas pre-estímulo, los grupos lesionados tardan más en recorrer la barra en comparación con los grupos control y Sham $\left({ }^{*} \mathbf{p}<0.05\right)$. A partir de los 120 días, los animales lesionados (6-OHDA) aumentan significativamente el tiempo, al compararlo con el grupo lesionado con tratamiento electromagnético (*p $<0.05$ y **p $<0.01$ a los 5 y 6 meses). Elaboración personal.
Los bioefectos de la estimulación electromagnética dependen de la frecuencia, la densidad de flujo y la duración de la exposición (Ni \& Chen, 2015). De acuerdo con la guía de la Comisión Internacional de Protección de Radiación no Ionizante, por sus siglas en inglés (ICNIRP) del 2010, el umbral de exposición a $60 \mathrm{~Hz}$, tanto para el público en general como el expuesto ocupacionalmente es de $20 \mathrm{mT}$ y $1 \mathrm{mT}$, respectivamente (ICNIRP, 2010). En este trabajo, la densidad de flujo utilizada se encuentra dentro del rango de algunos aparatos de magnetoterapia empleados en clínicas y hospitales (Belyaev et al., 2016; Mattsson \& Simko, 2019).

La estimulación electromagnética de $60 \mathrm{~Hz}$ mostró tener efectos benéficos en el modelo animal de la EP al aplicarla durante largo plazo. En particular, el tratamiento empleado no afectó el peso de los animales control y con falsa lesión, similar a lo previamente reportado por otros autores (Lee et al., 2006; Mahdavi, Sahraei, Rezaei-Tavirani \& Najafi, 2016). El porcentaje de aumento de peso en los animales lesionados expuestos al CEM sugiere al resultar similar al de los animales control, que el tratamiento electromagnético impide la pérdida de peso evidenciada en el modelo de EP. Esto pudo deberse a un aumento en la ingesta alimenticia inducida por la estimulación utilizada. El aumento en la ingesta inducido por la estimulación cerebral profunda ha sido observado tanto en ratas lesionadas con 6-OHDA (Guimarães et al., 2013), así como en pacientes con EP (Ruzika et al., 2012).

En el presente trabajo, la lesión correspondió a una de tipo moderada según estudios previos (Allbutt \& Henderson, 2007; Moreno-Fitz et al., 2015); es decir, sin pérdida total de las neuronas dopaminérgicas en el área inyectada con 6-OHDA. Reportes previos han propuesto que se necesita la existencia de neuronas dopaminérgicas funcionales en la SNc del modelo de hemiparkinson en rata, para que con

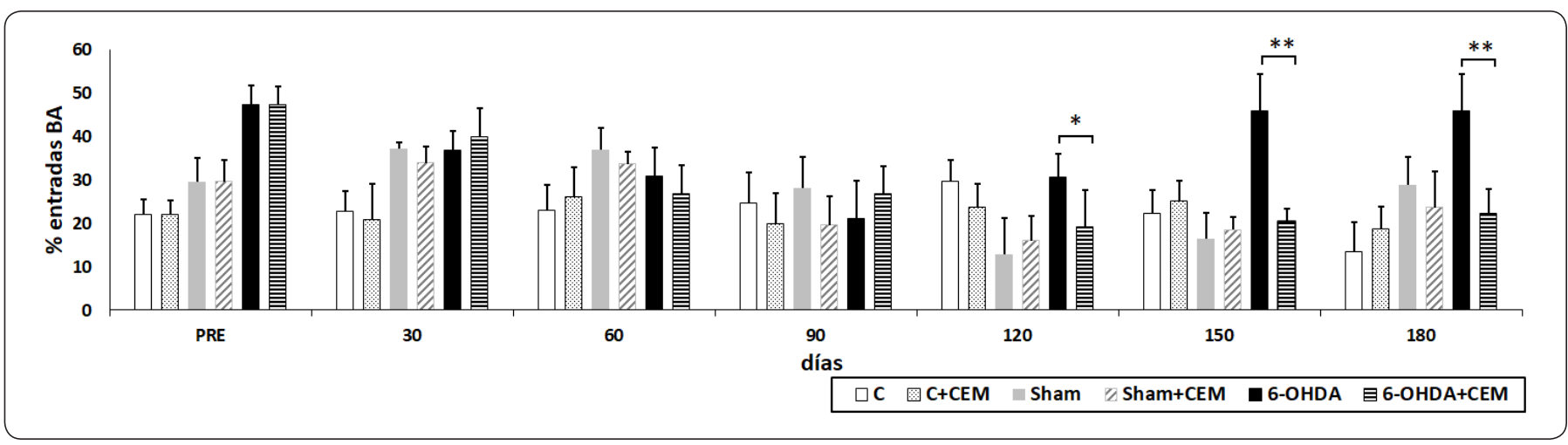

Figura 6. Laberinto Elevado en Cruz. Porcentaje \pm EEM en el laberinto de entradas a brazos abiertos, antes de iniciar la estimulación (pre), a los 30, 60, 90, 120, 150 y 180 días después de la estimulación con CEM. Durante las pruebas pre-estímulo, los grupos lesionados muestran un mayor porcentaje de entradas comparados con los grupos control y Sham $(* p<0.05)$. A los 4 meses se observa un aumento estadísticamente significativo de entradas a brazos abiertos en los animales lesionados respecto a los animales tratados con CEM (*p $<$ $0.05)$ incremento que continua a los 5 y 6 meses $(* p<0.01)$. Elaboración personal. 
Tabla II. Prueba de campo abierto.

\begin{tabular}{|c|c|c|c|c|c|c|c|}
\hline \multicolumn{8}{|c|}{ DISTANCIA RECORRIDA (cm) } \\
\hline Días & $\mathbf{C}$ & $\mathrm{C}+\mathrm{CEM}$ & Sham & Sham+CEM & 6-OHDA & 6-OHDA+CEM & Significancia \\
\hline pre & $3613 \pm 355$ & $3613 \pm 355$ & $3395 \pm 217$ & $3395 \pm 217$ & $3542 \pm 248$ & $3542 \pm 248$ & n.s. \\
\hline 60 & $3348 \pm 122$ & $2430 \pm 452$ & $3967 \pm 365$ & $2823 \pm 167$ & $3376 \pm 320$ & $3052 \pm 360$ & n.s. \\
\hline 120 & $2925+318$ & $1855 \pm 247^{*}$ & $2790 \pm 437$ & $1310 \pm 53^{*}$ & $1300 \pm 278^{*}$ & $1485 \pm 305^{*}$ & $* \mathrm{p}<0.05$ vs C y Sham \\
\hline 180 & $1923 \pm 331$ & $1271 \pm 488$ & $1285 \pm 420$ & $967 \pm 228^{*}$ & $810 \pm 107^{*}$ & $495 \pm 159 * * \#$ & $\begin{array}{c}* \mathrm{p}<0.05 \text { vs } \mathrm{C} \\
* * \mathrm{p}<0.01 \text { vs } \mathrm{C} \\
\# \mathrm{p}<0.05 \text { vs } 6 \text {-OHDA }\end{array}$ \\
\hline \multicolumn{8}{|c|}{ TOQUES A PAREDES } \\
\hline pre & $48 \pm 6.1$ & $48 \pm 6.1$ & $47 \pm 6.9$ & $47 \pm 6.9$ & $39 \pm 3.6$ & $39 \pm 3.6$ & n.s. \\
\hline 60 & $39 \pm 3.4$ & $28 \pm 4.6$ & $36 \pm 5.1$ & $27 \pm 7.4$ & $29 \pm 4.6$ & $8 \pm 6.5$ & n.s. \\
\hline 120 & $17 \pm 4.1$ & $15 \pm 2.3$ & $14 \pm 6.5$ & $10 \pm 5.6$ & $9 \pm 3.3$ & $10 \pm 1.1$ & n.s. \\
\hline 180 & $9 \pm 1.4$ & $11 \pm 2.1$ & $10 \pm 2.1$ & $8 \pm 2.5$ & $10 \pm 2.2$ & $4 \pm 2.3^{*}$ & $\begin{array}{c}* \mathrm{p}<0.05 \text { vs } \mathrm{C} y \\
\text { 6-OHDA }\end{array}$ \\
\hline
\end{tabular}

Promedio \pm EEM de la distancia recorrida $(\mathrm{cm})$, el número de cuadros externos y el número de toques a las paredes laterales, durante los 15 minutos de la prueba. Se observa una disminución tanto en los movimientos horizontales como verticales en todos los grupos a lo largo del experimento $\left({ }^{*} \mathrm{p}<0.05\right)$. ANOVA de dos vías con múltiples comparaciones. n.s. = no significativo.

la estimulación electromagnética se den mejorías motoras (Hsieh et al., 2015). Probablemente a los 6 meses las neuronas dopaminérgicas que persisten aún sean funcionales.

La estimulación con el CEM indujo a una mejoría en la prueba de giros inducidos, a pesar de que no cambió el número de neuronas dopaminérgicas en la SNc. Esto puede explicarse por otros mecanismos, como el aumento en los factores neurotróficos y la reducción del nivel oxidante, ambos como consecuencia de la estimulación magnética reportada para modelos animales de la enfermedad de Alzheimer (Akbarnejad et al., 2018) o de Huntington (Tasset et al., 2012).

Pocos son los estudios realizados a largo plazo utilizando estimulación electromagnética y con resultados reportados muy variables. La mayoría de los estudios han sido por periodos más cortos de estimulación y con animales control. Por ejemplo, la aplicación por 24 semanas de un CEM de $100 \mathrm{mT}$ y $50 \mathrm{~Hz}$, no tuvo ningún efecto en las pruebas de conducta y memoria de ratas macho (Lai et al., 2016).

Varios autores han señalado que la deficiencia dopaminérgica provoca un incremento de la demora, tanto para iniciar el movimiento, como para cruzar la barra de equilibrio y alcanzar la plataforma; correspondientes con la acinesia y bradicinesia observadas en modelos animales de la EP (Blanco et al., 2010; Truong et al., 2006). El tratamiento con el CEM utilizado en nuestro estudio, parece compensar esa deficiencia dopaminérgica y permitir mantener una mejoría en el equilibrio. Además, los tratamientos electromagnéticos en pacientes con la EP han mostrado una mejora en el temblor postural (Malling et al., 2019) y otros síntomas motores (Liu, Wang, He \& Ye, 2008).

Reportes sobre el efecto de la estimulación electromagnética en parámetros conductuales, muestran resultados contradictorios (Szemerszky et al., 2010; Viera et al., 2019). En nuestro trabajo, en los primeros 3-4 meses de tratamiento electromagnético no observamos cambios en las pruebas de campo abierto y laberinto en cruz. La lesión con 6-OHDA indujo a un aumento en el porcentaje de entradas a los brazos abiertos del laberinto elevado, como ya había sido observado por otros autores (Badstuebner, Gimsa, Weber, Tuchscherer \& Gimsa, 2017). Este parámetro se redujo en los últimos tres meses de tratamiento con el CEM en el modelo de la EP, alcanzando valores similares a los animales control.

Los tratamientos electromagnéticos en pacientes con EP reportan efectos antidepresivos (Liu et al., 2008). Sin embargo, en la prueba de campo abierto, el tratamiento electromagnético redujo tanto los movimientos horizontales como los verticales; lo que puede reflejar un deterioro en la locomoción y/o un fenómeno de habituación. Esta última opción fue propuesta por Badstuebner y colaboradores, quienes encontraron una disminución en la distancia recorrida después de 6 semanas de estimulación cerebral profunda en el modelo de la EP en rata (Badstuebner et al., 2017). En ratas intactas una exposición electromagnética continua durante 4-6 semanas indujo a conductas tipo depresivas (Szemerszky et al., 2010). En este caso, los autores proponen un efecto estresante, producto de la estimulación magnética aplicada en forma continua, que difiere de nuestro experimento en el 
que el CEM se aplicó durante dos horas al día. Sin embargo, es importante seguir explorando las opciones propuestas para dilucidar los resultados ansiolíticos y ansiogénicos observados en las pruebas de campo abierto y laberinto elevado.

El presente estudio presenta algunas limitaciones que deben de considerarse en futuros experimentos. 1) solo se usaron animales machos. El riesgo de desarrollar esta patología es dos veces mayor en los hombres que en las mujeres, pero las mujeres experimentan una progresión más rápida de la enfermedad y una tasa de supervivencia más baja (Cerri, Mus \& Blandini, 2019). Por lo tanto, es necesario elucidar el papel del sexo en la respuesta al tratamiento con CEM en el modelo animal de la EP, 2) no se exploraron los mecanismos moleculares subyacentes que expliquen el efecto benéfico observado, 3 ) las pruebas exploradas fueron más de tipo motor, pero es necesario probar otras relacionadas con la ansiedad; así como, aumentar el número de animales estudiados.

En conclusión, los principales resultados obtenidos apoyan el uso de la terapia electromagnética de $60 \mathrm{~Hz}$ como un posible tratamiento coadyuvante en problemas motores de pacientes con EP.

\section{Agradecimientos}

Los autores desean agradecer a la Sra. Antonia Rojas Terrazas por su ayuda en el cuidado de los animales.

\section{Apoyos}

Este trabajo fue financiado por la UNAM-DGAPA (IN214017 e IN220120).

\section{CONFLICTO DE INTERÉS}

Los autores declaran que no existe ningún conflicto de interés.

\section{REFERENCIAS}

Akbarnejad, Z., Esmaeilpour, K., Shabani, M., AsadiShekaari, M., Saeedi, M. \& Ahmadi-Zeidabadi, M. (2018). Spatial memory recovery in Alzheimer's rat model by electromagnetic field exposure. Int. J. Neurosc., 128(8), 691-696. DOI: 00207454.2017.1411353

Allbutt, H. \& Henderson, J. (2007). Use of the narrow beam test in the rat, 6-hydroxydopamine model of Parkinson's disease. J. Neurosc. Meth., 159, 195-202. DOI: 10.1016/j. jneumeth.2006.07.006.

Arias-Carrión, O., Verdugo-Díaz, L., Feria-Velasco, A., Millán-Aldaco, D., Gutiérrez, A., Hernández-Cruz, A. \& Drucker-Colin, R. (2004). Neurogenesis in the subventricular zone following transcranial magnetic field stimulation and nigrostriatal lesions. J. Neurosci. Res., 78, 16-28. DOI: 10.1002/jnr.20235.

Badstuebner, K., Gimsa, U., Weber, I., Tuchscherer, A. \& Gimsa, J. (2017). Deep Brain Stimulation of Hemiparkinsonian Rats with Unipolar and Bipolar
Electrodes for up to 6 Weeks: Behavioral Testing of Freely Moving Animals. Parkinson Dis., 5693589. DOI: $10.1155 / 2017 / 569358$.

Belyaev, I., Dean, A., Eger, H., Hubmann, G., Jandrisovits, R., Kern, M., Kundi, M., Moshammer, H., Lercher, P., Müller, K., Oberfeld, G., Ohnsorge, P., Pelzmann, P., Scheingraber, C. \& Thill, R. (2016). "EUROPAEM EMF Guideline 2016 for the prevention, diagnosis and treatment of EMF-related health problems and illnesses". Rev. Environ. Health, 31(3), 363-397. DOI: 10.1515/ reveh-2016-0011.

Blanco, L., Lorigados, L., Fernández, C., Serrano, T., Pavón, N. \& Francis, L. (2010). Aplicación del test de la barra transversal modificado para evaluar ratas Hemiparkinsonizadas. Acta Biológica Colombiana, 15(2), 189-201.

Chance, W., Grossman, C., Newrock, R., Bovin, G., Yerian, K., Schmitt, G. \& Mendenhall, C. (1995). Effects of electromagnetic fields and gender on neurotransmitters and amino acids in rats. Physiol. Behav., 58(4), 743-748. DOI: 10.1016/0031-9384(95)00090-6.

Cerri, S., Mus, L. \& Blandini, F. (2019). Parkinson's Disease in Women and Men: What's the Difference? J. Parkinsons Dis., 9(3), 501-515. DOI: 10.3233/JPD-191683.

Chervyakov, A., Chernyavsky, A., Sinitsyn, D. \& Piradov, M. (2015). Possible mechanisms underlying the therapeutic effects of transcranial magnetic stimulation. Front. Hum. Neurosci., 9, 303. DOI: 10.3389/fnhum.2015.00303.

Fàbregues, O., Gironell, A., Rosselló-Jiménez, D. \& Regueras, E. (2017). Unidades de trastornos del movimiento y tratamiento de las fluctuaciones motoras de la enfermedad de Parkinson avanzada. Rev. Neurol., 65(09), 396-404. DOI: 10.33588/rn.6509.2017106.

Gervasi, F., Murtas, R., Decarli, A. \& Russo, A. G. (2019). Residential distance from high-voltage overhead power lines and risk of Alzheimer's dementia and Parkinson's disease: a population-based case-control study in a metropolitan area of Northern Italy. Int. J. Epidemiol., Dec. 1, 48(6), 1949-1957.DOI: 10.1093/ije/dyz139.

Gómez-Chavarín, M., Diaz-Pérez, R., Morales-Espinoza, R., Fernandez-Ruis, J., Roldan-Roldan, G. \&, Torner, C. (2013). Efecto de la exposición al pesticida rotenona sobre el desarrollo del sistema dopaminérgico nigroestriatal en ratas. Salud Mental, 36(1), 1-8.

Guimarães, J., Moura, E., Silva, E., Aguiar, P., Garrett, C. \& Vieira-Coelho, M. A. (2013). Locus coeruleus is involved in weight loss in a rat model of Parkinson's disease: an effect reversed by deep brain stimulation. Brain Stimul., 6(6), 845-855. DOI: 10.1016/j.brs.2013.06.002.

Gunnarsson, L.G. \& Bodin, L. (2019). Occupational Exposures and Neurodegenerative Diseases-A Systematic Literature Review and Meta-Analyses. Int. J. Environ. Res. Public Health. Jan 26, 6(3), 337. DOI: 10.3390/ijerph16030337. 
Horii, Y., McTaggart, I. \& Kawaguchi, M. (2018). Testing Animal Anxiety in Rats: Effects of Open Arm Ledges and Closed Arm Wall Transparency in Elevated Plus Maze Test. J. Vis. Exp., Jun 29;(136), 56428. DOI: $10.3791 / 56428$.

Hsieh, T. H., Huang, Y. Z., Rotenberg, A., Pascual-Leone, A., Chiang, Y. H., Wang, J. Y. \& Chen, J. J. (2015). Functional Dopaminergic Neurons in Substantia Nigra are Required for Transcranial Magnetic Stimulation-Induced Motor Plasticity. Cereb. Cortex, 25(7), 1806-1814. DOI: 10.1093/cercor/bht421.

International Commission on Non-Ionizing Radiation Protection. (2010). Guidelines for limiting exposure to time-varying electric and magnetic fields $(1 \mathrm{~Hz}$ to 100 kHz). Health Phys., 99(6), 818-836. DOI: 10.1097/ HP.0b013e3181f06c86.

Jankovic, J. (2008). Parkinson's disease: clinical features and diagnosis. J. Neurol. Neurosurg. Psychiatr., 79(4), 368376. DOI: $10.1136 /$ jnnp.2007.131045.

Lai, J., Zhang, Y., Liu, X., Zhang, J., Ruan, G., Chaugai, S. \& Wang, D.W. (2016). Effects of extremely low frequency electromagnetic fields $(100 \mathrm{mT})$ on behaviors in rats. NeuroToxicology, 52, 104-113. DOI: 10.1016/j. neuro.2015.11.010.

Lee, H., Kim, S., Choi, S., Gimm, Y., Pack, J., Choi, H. D. \& Lee, Y. S. (2006). Long-term exposure of Sprague Dawley rats to $20 \mathrm{kHz}$ triangular magnetic fields. Int. J. Radiat. Biol., 82(4), 285-291. DOI: 10.1080/09553000600721809.

Lee, J., Kim, S., Ko, A-H., Lee, J., Yu, J., Seo, J., Cho, B. P. \& Cho, S-R. (2013). Therapeutic effects of repetitive transcranial magnetic stimulation in an animal model of Parkinson's disease. Brain Res., 1537, 290-302. DOI: 10.1016/j.brainres.2013.08.051.

Liu, T., Wang, S., He, L. \& Ye, K. (2008). Chronic exposure to low-intensity magnetic field improves acquisition and maintenance of memory. NeuroReport, 19(5), 549-552. DOI: 10.1097/WNR.0b013e3282f8b1a0.

Mahdavi, S., Sahraei, H., Rezaei-Tavirani, M. \& Najafi, A. (2016). Common behaviors alterations after extremely low-frequency electromagnetic field exposure in rat animal model. Electromagn. Biol. Med., 35(3), 222-227. DOI: $10.3109 / 15368378.2015 .1054401 \mathrm{https}: / /$ doi.org/1 $0.3109 / 15368378.2015 .105440$

Malling, A. S. B., Morberg, B. M., Wermuth, L., Gredal, O., Bech, P. \& Jensen, B. R. (2019). The effect of 8 weeks of treatment with transcranial pulsed electromagnetic fields on hand tremor and inter-hand coherence in persons with Parkinson's disease. J. Neuroeng. Rehabil., 16(1),19. DOI: 10.1186/s12984-019-0491-2.

Mattsson, M. O. \& Simko, M. (2019). "Emerging medical applications based on non-ionizing electromagnetic fields from $0 \mathrm{~Hz}$ to $10 \mathrm{THz}$." Med. Devices (Auckl), 12, 347368. DOI: 10.2147/MDER.S214152.

Miller, K. J., Suárez-Iglesias, D., Seijo-Martínez, M. \& Ayán,
C. (2020). Fisioterapia para la congelación de la marcha en la enfermedad de Parkinson: revisión sistemática y metaanálisis. Rev. Neurol., 70(05), 161-170. DOI: 10.33588/rn.7005.2019417.

Moreno-Fitz, J., Medina-Salazar, I., Chávez-Hernández, V., Elías-Viñas, D. \& Verdugo-Díaz, L. (2015). Efecto de la exposición a campos magnéticos de extrema baja frecuencia en un modelo de hemiparkinson en ratas. Eneurobiología, 7(12), 290615.

Ni, Z. \& Chen, R. (2015). Transcranial magnetic stimulation to understand pathophysiology and as potential treatment for neurodegenerative diseases. Transl. Neurodegener., 4, 22. DOI: $10.1186 / \mathrm{s} 40035-015-0045-x$.

Picazo, O., Chuc-Meza, E., Anaya-Martinez, V., Jimenez, I., Aceves, J. \& Garcia-Ramirez, M. (2009). 6-Hydroxydopamine lesion in thalamic reticular nucleus reduces anxiety behaviour in the rat. Behav. Brain Res., 197(2), 317-322. DOI: 10.1016/j.bbr.2008.08.047.

Paxinos, G. \& Watson, Ch. (2005). The Rat Brain, in stereotaxic coordinates, San Diego: Elsevier Academic Press, 5 ta ed.

Rektorová, L. \& Anderkova, I. (2017). Noninvasive Brain Stimulation and Implications for Nonmotor Symptoms in Parkinson's Disease. Int. Rev. Neurobiol., 134,1091-1110. DOI: 10.1016/bs.irn.2017.05.009.

Ruzika, F., Jech, R., Nova'kova, L., Urgos.i'k, D., Vymazal, J. \& Růžička, E. (2012) Weight Gain Is Associated with Medial Contact Site of Subthalamic Stimulation in Parkinson's Disease. PLoS ONE, 7(5), e38020. DOI: 10.1371/journal.pone.0038020.

Schmitt, U. \& Hiemke, C. (1998). Combination of open field and elevated plus-maze: a suitable test battery to assess strain as well as treatment differences in rat behavior. Prog. Neuro-Psychopharmacol. \& Biol. Psychiat., 22(7), 1197-1215. DOI: 10.1016/s0278-5846(98)00051-7.

Shukla, A. \& Vaillancourt, D. (2014). Treatment and Physiology in Parkinson's disease and Dystonia: Using TMS to Uncover the Mechanisms of Action. Curr. Neurol. Neurosci. Rep., 14(6), 449. DOI: 10.1007/s11910-0140449-5.

Shukla, A.W., Shuster, J. J., Chung, J. W., Vaillancourt, D E, Patten, C., Ostrem, J. \& Okun, M. S. (2016). Repetitive Transcranial Magnetic Stimulation (rTMS) Therapy in Parkinson Disease: A Meta-Analysis. PM R. Apr., 8(4), 356-366. DOI: 10.1016/j.pmrj.2015.08.009.

Szemerszky, R., Zelena, D., Barna, I. \& Bárdos, G. (2010). Stress-related endocrinological and psychopathological effects of short- and long-term $50 \mathrm{~Hz}$ electromagnetic field exposure in rats. Brain Res. Bull., 81(1), 92-99. DOI: 10.1016/j.brainresbull.2009.10.015.

Tasset, I., Medina, F. J., Jimena, I., Agüera, E., Gascón, F., Feijóo, M., Sánchez-López, F., Luque, E., Peña, J., Drucker-Colín, R. \& Túnez, I. (2012). Neuroprotective effects of extremely low-frequency 
electromagnetic fields on a Huntington's disease rat model: effects on neurotrophic factors and neuronal density. Neuroscience, 209, 54-63. DOI: 10.1016/j. neuroscience.2012.02.034.

Truong, L., Allbutt, H., Kassiou, M. \& Henderson, J. M. (2006). Developing a preclinical model of Parkinson's disease: a study of behaviour in rats with graded 6-OHDA lesions. Behav. Brain Res., 169, 1-9. DOI: 10.1016/j. bbr.2005.11.026.

Umarao, P., Bose, S., Bhattacharyya, S., Kumar, A. \& Jain, S. (2016). Neuroprotective Potential of Superparamagnetic
Iron Oxide Nanoparticles Along with Exposure to Electromagnetic Field in 6-OHDA Rat Model of Parkinson's Disease. J. Nanosci. Nanotechnol., 16(1), 261-269. DOI: 10.1166/jnn.2016.11103.

Vieira, J. C. F., Bassani, T. B., Santiago, R. M., de O Guaita, G., Zanoeli, J. M., daCunha, C. \& Vital, M. A. (2019). Anxiety-like behavior induced by 6-OHDA animal model of Parkinson's disease may be related to a dysregulation of neurotransmitter systems in brain areas related to anxiety. Behav. Brain Res., 371, 111981. DOI: 10.1016/j. bbr.2019.111981. 